\title{
LAS RELACIONES COMERCIALES EN AMÉRICA LATINA EN 1995 y 2015: UN ANÁLISIS DE REDES COMPLEJAS*
}

Recibido: 10 de mayo de 2017 - Aprobado: 22 de agosto de 2017

DOI: $10.22395 /$ seec.v20n45a6

\author{
Luz Yadira Gómez Hernández ${ }^{* *}$ \\ Andrey David Ramos Ramírez ${ }^{* * *}$
}

\section{RESUMEN}

El objetivo de este trabajo es analizar las relaciones comerciales en América Latina en los años 1995 y 2015 utilizando el enfoque de redes complejas, con datos de los cinco primeros socios de importaciones y exportaciones de 17 países de la región. El estudio de las propiedades del grafo se complementa con el análisis de hechos históricos importantes y la composición de las canastas de importaciones y exportaciones. Los resultados muestran la tendencia en la región a comerciar con economías grandes en Asia y América del Norte a las que se les exporta principalmente bienes primarios y desde las que se importa tecnología, bienes industriales y de capital.

\section{PALABRAS CLAVE}

Comercio internacional; política comercial; globalización; integración económica; redes complejas; América Latina.

\section{CLASIFICACIÓN JEL}

$\mathrm{F} 13, \mathrm{~F} 14, \mathrm{O} 24$

\section{CONTENIDO}

Introducción; 1. Metodología; 2. Redes de exportaciones e importaciones en América Latina, 1995 y 2015; 3. Comentarios Finales; Bibliografía.

\footnotetext{
Este artículo de investigación surge como resultado del trabajo realizado en el curso "Crisis Macroeconómicas y Sistemas Complejos" del programa de Maestría en Economía de la Universidad de Buenos Aires, dictado por los profesores Daniel Heymann, Roberto Perazzo y Viktoriya Semeshenko.

** Economista, Universidad Nacional de Colombia, Medellín, Colombia. Magíster en Economía, Universidad de Buenos Aires, Buenos Aires, Argentina. Docente Microeconomía II, Universidad Nacional de Colombia, Medellín, Colombia. Miembro del grupo de investigación Economía, Cultura y Políticas (Clasificado por Colciencias en Categoría C en 2015). Dirección: Calle 59A \# 63-20, Medellín, Colombia. Correo electrónico: lygomezh@unal. edu.co.

***Economista, Universidad Nacional de Colombia, Medellín. Estudiante de la Maestría en Ciencias Económicas, Universidad Nacional de Colombia, Medellín, Colombia. Docente Econometría I, Universidad Nacional de Colombia, Medellín, Colombia. Miembro del grupo de investigación Economía, Cultura y Políticas (Clasificado por Colciencias en Categoría C en 2015). Dirección: Calle 59A \# 63-20, Medellín, Colombia. Correo electrónico: adramosr@unal.edu.co.
} 


\title{
TRADE RELATIONS IN LATIN AMERICA IN 1995 AND 2015: AN ANALYSIS OF COMPLEX NETWORKS
}

\begin{abstract}
The objective of this work is to analyze trade relations in Latin America in the years 1995 and 2015 using the approach of complex networks, with data from the first five imports and exports partners from 17 countries in the region. The study of the properties of the network is complemented by the analysis of important historical facts and the composition of import and export baskets. The results show the region's tendency to trade with large economies in Asia and North America, where it exports mainly primary goods and from which technology, industrial and capital goods are imported.
\end{abstract}

\section{KEYWORDS}

International trade; trade policy; globalization; economic integration; complex networks; Latin America.

\section{JEL CLASSIFICATION}

F13, F14, O24

\section{CONTENT}

Introduction; 1. Methodology; 2. Export and import networks in Latin America, 1995 and 2015; 3. Concluding Comments; Bibliography.

\section{AS RELAÇÕES COMERCIAIS NA AMÉRICA LATINA EM 1995 E 2015: UMA ANÁLISE DE REDES COMPLEXAS}

\section{RESUMO}

O objetivo deste trabalho é analisar as relações comerciais na América Latina nos anos 1995 e 2015, utilizando a abordagem de redes complexas, com dados dos cinco primeiros sócios de importações e exportações de 17 países da região. O estudo das propriedades do grafo se complementa com a análise de fatos históricos importantes e da composição de cestas de importações e exportações. Os resultados mostram a tendência na região de comercializar com economias grandes na Ásia e na América do Norte, regiões às quais se exportam principalmente bens primários e das quais se importam tecnologia, bens industriais e de capital.

\section{PALAVRAS-CHAVE}

Comércio internacional; política comercial; globalização; integração econômica; redes complexas; América Latina.

\section{CLASSIFICAÇÃO JEL}

$\mathrm{F} 13, \mathrm{~F} 14, \mathrm{O} 24$

\section{CONTEÚDO}

Introdução; 1. Metodologia; 2. Redes de exportações e importações na América Latina, 1995 e 2015; 3. Comentários finais; Bibliografia. 


\section{INTRODUCCIÓN}

En los sistemas complejos el acoplamiento de un número de elementos, cuyo comportamiento individual responde a leyes sencillas, puede generar conductas grupales complicadas. Como lo plantean Heymann, Perazzo y Zimmerman (2013), esta característica se asocia con propiedades de no-linealidad que pueden explicar por qué algunos conjuntos no constituyen ampliaciones proporcionales de sus componentes. Este es el caso de la economía, que al ser un sistema compuesto por entidades e individuos interconectados con patrones macro no-lineales, abarca fenómenos difíciles de explicar con las herramientas econométricas tradicionales, pero que pueden ser estudiados por medio de sistemas complejos que permitan modelar aspectos como el efecto de los otros sobre el desempeño y la evolución del sistema. Según los autores, una expresión temprana de estos sistemas es la asociación entre división del trabajo y tamaño del mercado, formulada por Adam Smith.

Las redes son unas de las herramientas de sistemas complejos más utilizadas para caracterizar de forma matemática aquellos sistemas sociales, económicos o biológicos, en los cuales los vínculos y las interacciones entre los individuos juegan un papel central en su desempeño y evolución. Heymann, Perazzo y Zimmerman (2013) y Zhang et al. (2017) definen una red como una entidad compuesta por un conjunto finito de nodos que pueden representar objetos de distinta naturaleza y que están conectados entre sí a través de relaciones o vínculos. Cuando la red tiene características generales de un sistema complejo como organización jerárquica, capacidad de adaptación y simplificación de los componentes y de sus interacciones, se habla entonces de una red compleja (Serrano y Boguña, 2003).

El análisis de redes complejas es aplicado con frecuencia en estudios empíricos sobre comercio internacional en la construcción de la Red de Comercio Mundial (RCM), como en los trabajos de Serrano y Boguña (2003), Li, Jin y Cheng (2003), Garlaschelli y Loffredo (2005), Kastelle, Steen y Liesch (2006), Serrano, Boguña y Vespignan (2007), entre otros. Como plantean Fagiolo, Reyes y Schiavo (2008), en estos análisis las relaciones comerciales se representan mediante una red, en la cual los países juegan el papel de nodos y un vínculo describe la presencia de una relación de importación o exportación entre ellos.

El presente trabajo tiene como objetivo estudiar la evolución de las relaciones de comercio de 17 países latinoamericanos desde un enfoque de redes complejas. Utilizando datos de los cinco primeros socios comerciales en importaciones y exportaciones en 1995 y 2015, se construyen las redes y se analiza el cambio en sus propiedades. Las redes son ponderadas por el volumen de los flujos comerciales para estudiar con mayor profundidad la posible heterogeneidad entre las relaciones 
y entender la importancia de cada nodo, dada su ubicación en el grafo. El análisis de las propiedades topológicas se complementa con información sobre la composición de las exportaciones e importaciones, datos sobre los acuerdos comerciales que se han establecido en la región y los cambios más importantes en la economía mundial para comprender los patrones de comercio observados.

El dominio temporal de la investigación fue elegido teniendo en cuenta los cambios en el panorama económico de América Latina en la etapa posterior a la adopción de los postulados del Consenso de Washington; en específico, mientras algunos países experimentaron procesos de apertura y liberalización económi$\mathrm{ca}$, otros adoptaron regímenes políticos que restringieron las relaciones comerciales con el resto del mundo. Estudiar 1995 y 2015 permite identificar los factores determinantes de los cambios en la trayectoria de desarrollo, generados por el comercio internacional y las principales falencias de las políticas comerciales para sortearlos. Los principales resultados muestran que durante el periodo de análisis, el principal cambio en la estructura de las redes de importaciones y exportaciones es el posicionamiento de China como socio comercial para todos los países de la región. Además, a pesar de los múltiples acuerdos intrarregionales que se han establecido en los últimos años no se han podido consolidar bloques comerciales fuertes.

Representar los flujos de comercio desde el enfoque de redes complejas tiene la ventaja con respecto a los modelos gravitacionales tradicionalmente elegidos, de centrarse en las relaciones subyacentes entre los países que conforman la red y entender la importancia de cada uno en la estructura. Según Bhattacharya et al. (2008), Fagiolo, Reyes y Schiavo (2008) y De Benedictis y Tajoli (2011), esto hace posible capturar los elementos que caracterizan la relación comercial entre dos países y las consecuencias que tienen el comportamiento y las relaciones directas e indirectas sobre toda la estructura de la red. Esto se conoce como el efecto de los otros y permite estudiar fenómenos como la transmisión de shocks económicos, el contagio de crisis y el cambio en el panorama económico mundial.

A pesar de sus ventajas, el estudio de las relaciones comerciales utilizando redes apareció apenas en la primera mitad del siglo pasado, y su desarrollo se dio de manera lenta y fragmentada. El primer trabajo encontrado es el de Hildgerdt (1943) quien construyó un grafo para representar las relaciones comerciales antes de la Segunda Guerra Mundial con el objetivo de orientar las políticas de impulso al comercio en la sociedad de naciones. El autor considera que las políticas comerciales formuladas por un país tienen efectos sobre sus socios directos e indirectos y, por tanto, la forma natural de estudiar las relaciones comerciales es a través de redes. 
Más adelante, en la década de 1990 las aplicaciones de redes a los flujos de comercio se enfocaron en las estadísticas descriptivas de la red para explicar la posición de los países y determinar la existencia de asimetrías en los flujos de comercio. Smith y White (1992) analizaron los flujos de commodities entre 1965 y 1980, caracterizaron la estructura de la red y contribuyeron a la literatura en dos aspectos: definiendo una medida de equivalencia para describir la preponderancia de los países que permitió clasificarlos en centro, semi-periferia y periferia; e introduciendo un elemento dinámico al estudiar las redes en más de un momento del tiempo (1965, 1970 y 1980).

Las contribuciones después del 2000 se enfocan en entender las características inherentes al sistema complejo y no solo en definir medidas para clasificar a los países. Serrano y Boguña (2003) construyen la RCM y encuentran en el grafo las características típicas de una red compleja' ${ }^{1}$, entre ellas: i) un grado de distribución libre de escala que implica un alto nivel de heterogeneidad entre los nodos; ii) la propiedad de mundo pequeño, que implica que la longitud del camino promedio entre cada par de vértices crece logarítmicamente con el tamaño del sistema y iii) un alto coeficiente de clustering, esto es, que los vecinos de determinado vértice tienen una alta probabilidad de estar conectados. Luego, Garlaschelli y Loffredo (2005) y Kali y Reyes (2007) construyeron la RCM como una red dirigida y confirmaron la marcada estructura jerárquica y la propiedad libre de escala.

De acuerdo con Vega (2007), Kali y Reyes (2007) y Bhattacharya et al. (2008), en términos económicos que una red sea compleja significa que la distribución de las conexiones entre los nodos no es un proceso aleatorio, sino que los efectos de la competencia y la cooperación materializada a través de acuerdos comerciales, así como las características diferenciales como el Producto Interno Bruto (PIB), el tamaño del mercado o el crecimiento económico determinan una estructura jerárquica en la cual es posible identificar un pequeño grupo de países que controlan gran parte de los flujos comerciales.

Estas investigaciones despertaron interés en modelar la heterogeneidad entre los países considerando información sobre las características de cada nodo y la relación comercial en la definición de las conexiones. Serrano y Boguña (2003), Fagiolo, Reyes y Schiavo (2007), Fagiolo, Reyes y Schiavo (2008) y De Benedictis y Tajoli (2011) adoptan un enfoque de redes ponderadas en las cuales cada vínculo es ponderado por una variable como el volumen de los flujos comerciales que aproxima la intensidad del comercio. Estos trabajos encuentran un alto grado de hetero-

Una descripción completa de esas características se puede ver en Heymann, Perazzo y Zimmerman (2013, p.206). 
geneidad no solo entre países, sino también en las características de los flujos de comercio. Además, los países que tienen conexiones más débiles tienden a estar conectados con países que juegan un papel de hubs. Esto también se puede asociar con las relaciones de centro-periferia que se estudian en la teoría de comercio i nternacional.

El artículo está organizado de la siguiente forma: en el segundo apartado se presentan los datos, se define una red compleja y sus características topológicas. En el tercero se analizan las redes de importaciones y exportaciones en 1995 y 2015, y los cambios más importantes a la luz de los hechos históricos, las tendencias y las políticas comerciales de estas dos décadas. En la última sección, se presentan los comentarios finales y las perspectivas de investigación utilizando redes complejas.

\section{METODOLOGÍA}

\subsection{Países objeto de estudio}

Para alcanzar el objetivo de estudiar la evolución de las relaciones de comercio en América Latina fueron tomados 17 países en los que se concentró la mayor parte de las exportaciones e importaciones en 1995 y 2015; por la baja magnitud de sus flujos comerciales no se incluyeron los países del Caribe, y Venezuela, Guyana y Surinam no fueron incluidos por falta de datos. Los 17 países aparecen en todas las redes, mientras que los países fuera de la región aparecen solo cuando son uno de los cinco primeros socios comerciales de algún país en América Latina; Venezuela, por su parte, aparece en las redes por ser socio comercial de los países analizados. Las gráficas presentan a cada país como un nodo con una etiqueta que corresponde a su código ISO².

La información sobre los flujos de importaciones y exportaciones, así como el PIB a precios corrientes provienen de la base World Integrated Trade Solution (WITS) del Banco Mundial, lo que garantiza la comparabilidad de los datos. Estas cifras están en millones de dólares corrientes y no han sido detalladas por tipos de productos. Los datos de los tratados comerciales vigentes en cada país latinoamericano, resumidos en el anexo, fueron tomados del Sistema de Información sobre Comercio Exterior (SICE) y de la Asociación Latinoamericana de Integración (ALADI) ${ }^{3}$.

\footnotetext{
2 Estándar que proporciona códigos para los nombres de países y otras dependencias administrativas.

3 Todos los datos utilizados en el trabajo están disponibles y pueden ser solicitados a los correos electrónicos de los autores lygomezh@unal.edu.co o adramosr@unal.edu.co.
} 
Tabla 1. Países objeto del análisis

\begin{tabular}{|c|c|c|c|c|c|}
\hline \multicolumn{6}{|c|}{ Países de América Latina } \\
\hline País & Código ISO & País & Código ISO & País & Código ISO \\
\hline México & MEX & Costa Rica & CRI & Bolivia & $\mathrm{BOL}$ \\
\hline Belice & BLZ & Panamá & PAN & Chile & $\mathrm{CHL}$ \\
\hline Guatemala & GTM & Colombia & $\mathrm{COL}$ & Argentina & ARG \\
\hline Honduras & HND & Venezuela & $\mathrm{VEN}^{4}$ & Uruguay & URY \\
\hline El Salvador & SLV & Ecuador & ECU & Paraguay & PRY \\
\hline Nicaragua & $\mathrm{NIC}$ & Perú & PER & Brasil & BRA \\
\hline \multicolumn{6}{|c|}{ Países del resto del mundo } \\
\hline País & Código ISO & País & Código ISO & País & Código ISO \\
\hline Estados Unidos & USA & Canadá & CAN & Japón & JPN \\
\hline Renio Unido & UK & Alemania & DEU & Italia & ITA \\
\hline China & $\mathrm{CHN}$ & Corea del Sur & KOR & España & ESP \\
\hline Países Bajos & NLD & Malasia & MYS & Nigeria & NIG \\
\hline $\begin{array}{l}\text { Antillas } \\
\text { Holandesas }\end{array}$ & ANT & Suiza & $\mathrm{CHE}$ & Bélgica & BLX \\
\hline Suecia & SWE & $\begin{array}{l}\text { Trinidad y } \\
\text { Tobago }\end{array}$ & TTO & Rusia & RUS \\
\hline Irlanda & IRL & India & IND & Vietnam & VIE \\
\hline
\end{tabular}

Fuente: elaboración propia con base en datos de WITS

Las redes fueron construidas con el software libre Gephi 0.9.1 de los autores Bastian, Heymann y Jacomy (2009). El posicionamiento de cada nodo fue determinado siguiendo la distribución propuesta por Fruchterman y Reingold (1991) que ubica al nodo más central en medio del grafo, permitiendo visualizar a los países más importantes para el comercio de la región.

\subsection{Definición de las redes de comercio internacional}

Una red $\mathcal{N}=(\mathcal{V}, \mathcal{L}, \mathcal{W}, \mathcal{P})$ está compuesta por un grafo $\mathcal{G}(\mathcal{V}, \mathcal{L})$, donde $\mathcal{V}=\{1,2, \ldots \ldots, n)$ es el conjunto de vértices o nodos (países presentados en la Tabla 1) y $\mathcal{L}=\{0,1, \ldots m\}$ es el conjunto de vínculos o aristas que los conectan. En grafos simples, $\mathcal{L}$ es una variable binaria, es decir $\mathcal{L}_{i j}=\{0,1\}$, donde $\mathcal{L}_{i j}$ toma el valor de 1 si entre los nodos $i$ y j existe una relación y 0 en otro caso, como plantean Jackson (2008), y De

\footnotetext{
4 Por no contar con información suficiente, solo aparece en la red como socio de otros países de la región.
} 
Benedectis y Tajoli (2011). En las redes construidas, la relación entre cada par de nodos se establece si un país se encuentra entre los primeros 5 socios comerciales en exportaciones e importaciones.

La segunda parte de la red $\mathcal{N}$ incluye información adicional acerca de las características relevantes de los vínculos $\mathcal{W}$ como el volumen de exportaciones e importaciones, y de los nodos $\mathcal{P}$ como PIB, tasa de crecimiento, población, localización geográfica. Según indican Fagiolo, Reyes y Schiavo (2008) y Jackson 2008), los elementos positivos en $\mathcal{W}$ actúan como ponderadores en la red, modificando su estructura y transformando las relaciones binarias simples en relaciones ponderadas que dan cuenta de la fortaleza de las conexiones. Esta información permite llevar a cabo un análisis estructural más completo de las relaciones comerciales e inferir los factores que explican la posible heterogeneidad y la mejor ubicación en la red. En este trabajo las relaciones son ponderadas por el volumen de exportaciones e importaciones. Las matrices utilizadas no son simétricas, pues existen relaciones no bidireccionales debido a que los vínculos van desde el país exportador hacia su socio comercial, sin que esto implique una relación en la dirección opuesta.

\subsection{PROPIEDADES TOPOLÓGICAS DE LAS REDES}

En el análisis de redes complejas se estudian algunas medidas asociadas a los nodos y al grafo conocidas como propiedades topológicas, que son empleadas para describir la estructura de la red y el posicionamiento de los nodos. En el caso específico de los flujos de comercio internacional, las propiedades topológicas arrojan información relevante para identificar los países más importantes como socios comerciales de una región y los factores que explican su posicionamiento central, al tiempo que permite caracterizar los patrones de comercio y el fortalecimiento de las relaciones comerciales en el interior de una región. Esto se logra a través de estadísticas descriptivas que permiten medir la conectividad y la centralidad o importancia de los nodos.

La noción más básica de la conectividad de un nodo es la vecindad, el número de nodos con los cuales se relaciona de forma directa o indirecta. Formalmente, para un nodo $i$, la vecindad corresponde al subconjunto de nodos $d \in \mathcal{V}$ tales que $\mathcal{L}_{i, d} \neq 0$; mientras que el grado $k_{i}$ es el tamaño de la vecindad del nodo, es decir, el número de conexiones o vecinos. Siguiendo a Heymann, Perazzo y Zimmerman (2013), la fracción de vecinos de un nodo que son, a su vez, vecinos entre sí se conoce como coeficiente de Clustering $\mathrm{C}_{i}$ y se define como:

$$
C_{i}=\frac{E_{i}}{k_{i}\left(k_{i}-1\right) / 2}
$$


Donde $E_{i}$ es el número de relaciones o vínculos que unen entre sí a los vecinos del nodo. El promedio del coeficiente de clustering para todos los nodos se conoce como Clustering medio e indica qué tan compacta es una red.

En redes dirigidas como las que se construyen en este trabajo, existen dos medidas relacionadas con el grado que permiten describir la importancia de los nodos: el grado de entrada y el grado de salida. El grado de entrada es el número de vínculos dirigidos que tiene como destino el nodo de interés; en este caso el grado de entrada de un país $i$ en la red de exportaciones es el número de países para los cuales este es uno de los 5 primeros destinos de exportaciones. El grado de salida es el número de vínculos que parten del nodo de interés; en la red de importaciones, el grado de salida del país $i$ es el número de países de los cuales este es uno de los primeros 5 socios comerciales como origen de las importaciones. En la red de exportaciones el tamaño de los nodos está dado por el grado de entrada, y en la red de importaciones, por el grado de salida.

En una red dirigida y ponderada, no solo es de interés el grado del nodo sino también el grado ponderado por pesos. Esta medida pondera el número de conexiones binarias del nodo teniendo en cuenta la variable o característica con las cuales se ponderan los vínculos y permiten establecer una medida más robusta de la relevancia del nodo en la red. En esta investigación, debido a que la red se pondera por el valor monetario de los flujos de comercio, el grado ponderado es una medida acertada que permite entender mejor la importancia del nodo y complementa al grado de entrada o de salida.

La posición de cada nodo en la red se mide en términos de la centralidad; siguiendo a Jackson (2008) y De Benedectis y Tajoli (2011) el grado estandarizado de centralidad $\varsigma_{i}^{d}$ de un vértice se calcula como el cociente entre el grado del nodo y el grado máximo posible dado por el número de nodos en la red menos $1(\mathrm{~N}-1)$. Es decir:

$$
\varsigma_{i}^{d}=\frac{\kappa_{i}}{\mathrm{~N}-1}
$$

Por extensión, el máximo nivel alcanzable de centralización se conoce como grado de centralización. Si $\varsigma_{i}^{d^{*}}$ es la centralidad del vértice que tiene la máxima centralidad, la varianza en los grados de los vértices se define como la suma de las diferencias absolutas entre la centralidad de cada nodo y la centralidad máxima. Formalmente, el grado de centralización de la red está dado por

$$
\varsigma^{d}=\frac{\sum_{i=1}^{\mathrm{N}}\left|\varsigma_{i}^{d}-\varsigma_{i}^{d^{*}}\right|}{(\mathrm{N}-2)(\mathrm{N}-1)}
$$


Otras medidas se enfocan en caracterizar las propiedades estructurales de la red en general, como es el caso de la distancia, el diámetro, la longitud del camino promedio y la densidad. La distancia $d_{i, j}$ entre los nodos $i$ y $j$, es el número mínimo de vínculos o conexiones que es necesario recorrer para unir ambos nodos; mientras que el diámetro se define como la distancia del camino entre todos los nodos más alejados en el grafo.

El promedio de las distancias entre cada par de nodos se define como la longitud del camino promedio:

$$
\mathrm{L}=\frac{1}{\mathrm{~N}(\mathrm{~N}-1)} \sum_{i, j, i \neq j} d_{i, j}
$$

A menor longitud del camino promedio, es posible afirmar que el grafo está mejor conectado y las relaciones entre los nodos son más estrechas. Por su parte, la densidad $\gamma$ permite medir qué tan cerca está una red de ser completa, es decir, de establecer todos los vínculos posibles entre los nodos. Si se define $m_{\max }$ como el número de vínculos de una red completa y $m$ como el número de vínculos de la red, la densidad está dada por la expresión (5).

$$
\gamma=\frac{m}{m_{\max }}
$$

\section{REDES DE EXPORTACIONES E IMPORTACIONES EN AMÉRICA LATINA, $1995 \mathrm{Y}$ 2015}

\subsection{Red de exportaciones}

Las propiedades topológicas de la red de exportaciones de América Latina en los años 1995 y 2015 se presentan en la tabla 2. Los resultados muestran que entre los dos años analizados el tamaño de la red se expandió, el diámetro y la longitud del camino promedio incrementaron, mientras que la densidad y el coeficiente de clustering promedio disminuyeron. En 1995 la distancia entre los nodos más alejados del grafo era de 5 pasos, medida que aumentó a 8 en 2015; además, en este año se estableció el 8,6\% de todas las posibles conexiones, medida que solo alcanzó el 6,7\% en 2015. Estos resultados indican que la red de exportaciones se expandió durante el período de análisis, lo que implica una menor conectividad entre los nodos en 2015 y unas relaciones comerciales menos estrechas. 
Las relaciones comerciales en América Latina en 1995 y 2015: un análisis de redes complejas

Tabla 2. Estadísticas descriptivas de las redes de exportaciones en 1995 y 2015

\begin{tabular}{|l|c|c|}
\hline \multicolumn{1}{|c|}{ Medida } & 1995 & 2015 \\
\hline Número de nodos & 32 & 36 \\
\hline Diámetro de la red & 5 & 8 \\
\hline Longitud del camino promedio & 1,792 & 2,685 \\
\hline Densidad del grafo & 0,086 & 0,067 \\
\hline Grado promedio & 5,312 & 4,722 \\
\hline $\begin{array}{l}\text { Grado ponderado con pesos (valores de las exporta- } \\
\text { ciones en millones de dólares) }\end{array}$ & $4.064,291$ & $15.784,833$ \\
\hline Coeficiente de clustering promedio & 0,149 & 0,123 \\
\hline
\end{tabular}

Fuente: elaboración propia utilizando Gephi 0.9.1

El análisis de las características topológicas de cada nodo muestra que en 1995 los dos principales socios de exportaciones de la región eran Estados Unidos, destino importante de las exportaciones de los 17 países del análisis, y Alemania, destino de las exportaciones de 11 países; no obstante, en términos monetarios eran Estados Unidos y Brasil quienes recibían el mayor valor de las exportaciones, siendo el flujo recibido por Brasil 10 veces menor que el de Estados Unidos. En el año 2015 el grado de entrada de Estados Unidos se redujo a 16, pues dejó de ser uno de los principales socios de Paraguay; sin embargo, sigue siendo el socio comercial más importante como destino de las exportaciones de la región. Un cambio a destacar en la red es la importancia ganada por China. Entre 1995 y 2015 el gigante asiático pasó de ser uno de los primeros 5 socios de las exportaciones de Perú y Uruguay, a ser destino importante de los productos de 9 países en la región, principalmente los sudamericanos, Panamá y México.

En el análisis de las estadísticas individuales de los nodos, el grado de entrada muestra que la mayor parte de las exportaciones de la región en 1995 y 2015 se envió hacia países en los cuales el mercado es amplio y el consumo final elevado. El coeficiente de correlación entre el PIB y el grado de entrada en la red de exportaciones valida este hallazgo; según los cálculos, en 1995 este coeficiente fue de 0,85 mientras que en 2015 fue de 0,88. Es interesante observar que a pesar de la tendencia de la región a intercambiar con economías grandes, Japón, Alemania y Reino Unido perdieron centralidad.

En las redes de exportaciones de 1995 y 2015, presentadas en el gráfico 1, se pueden visualizar los cambios en las relaciones comerciales. Se asignó un color claro a los países latinoamericanos objeto de análisis y un color oscuro a los socios 
comerciales por fuera de la región. Considerando que un país exportador puede ser también destino de las exportaciones, el tamaño de los nodos fue determinado de acuerdo con el grado de entrada, de modo que los más grandes son aquellos que reciben un mayor flujo de exportaciones desde la región. El tamaño de las aristas está determinado por el volumen de las exportaciones lo cual permite identificar cuáles son los flujos de mayor peso.

El número de nodos en la red se incrementó de 32 a 36, esto implica que el número de participantes externos en las exportaciones de la región se incrementó (de 15 a 19) como resultado del fortalecimiento de las relaciones comerciales con otras regiones (ver anexo). No obstante, se evidencia en el grafo que el cambio más drástico es el posicionamiento de China como socio estratégico de América Latina: mientras en 1995 era un nodo ubicado en la periferia, en 2015 tanto su tamaño como su centralidad se incrementaron significativamente. El posicionamiento del país asiático en la red estuvo acompañado de la disminución de importancia de los países de la Unión Europea (Alemania, España, Italia) y Japón que eran centrales en 1995 y que se desplazaron hacia la periferia del gráfico para 2015.

\subsection{Red de importaciones}

La evolución de la red de importaciones de América Latina durante el período de análisis muestra un panorama diferente a las exportaciones. Como se observa en la tabla 3, en 2015 el tamaño de la red se redujo y el grado promedio y coeficiente de Clustering se incrementaron. Mientras que en 1995 el tamaño de la red fue de 29 nodos es decir, participaban 12 países externos a la región, en 2005 este número se redujo a $26^{5}$. La medida de la densidad indica que en 1995 se había establecido solo el 10,5\% de todas las posibles conexiones entre los nodos, porcentaje que ascendió al 13,1\% en 2015. Esto evidencia que durante el período de análisis, los vínculos comerciales de importaciones de los países latinoamericanos se volvieron más estrechos, es decir, las relaciones intrarregionales se fortalecieron y la demanda por bienes y servicios producidos dentro de la región se incrementó. Sin embargo, Belice, Panamá, Ecuador, Chile, Bolivia, Uruguay y Paraguay no son principales socios de las importaciones de ningún otro país.

El análisis de las características topológicas de cada nodo indica que en 1995 la mayor cantidad de importaciones de la región provenía de Estados Unidos, Japón y Guatemala; no obstante, al estudiar el grado de salida ponderado de cada nodo se observa que la región compraba ocho veces más a Estados Unidos que a

La diferencia entre el número de nodos de la red de importaciones y la red de exportaciones está determinada por el número de países externos a la región que intervienen en las relaciones comerciales. 
Gráfico 1. Red de exportaciones 1995 y 2015

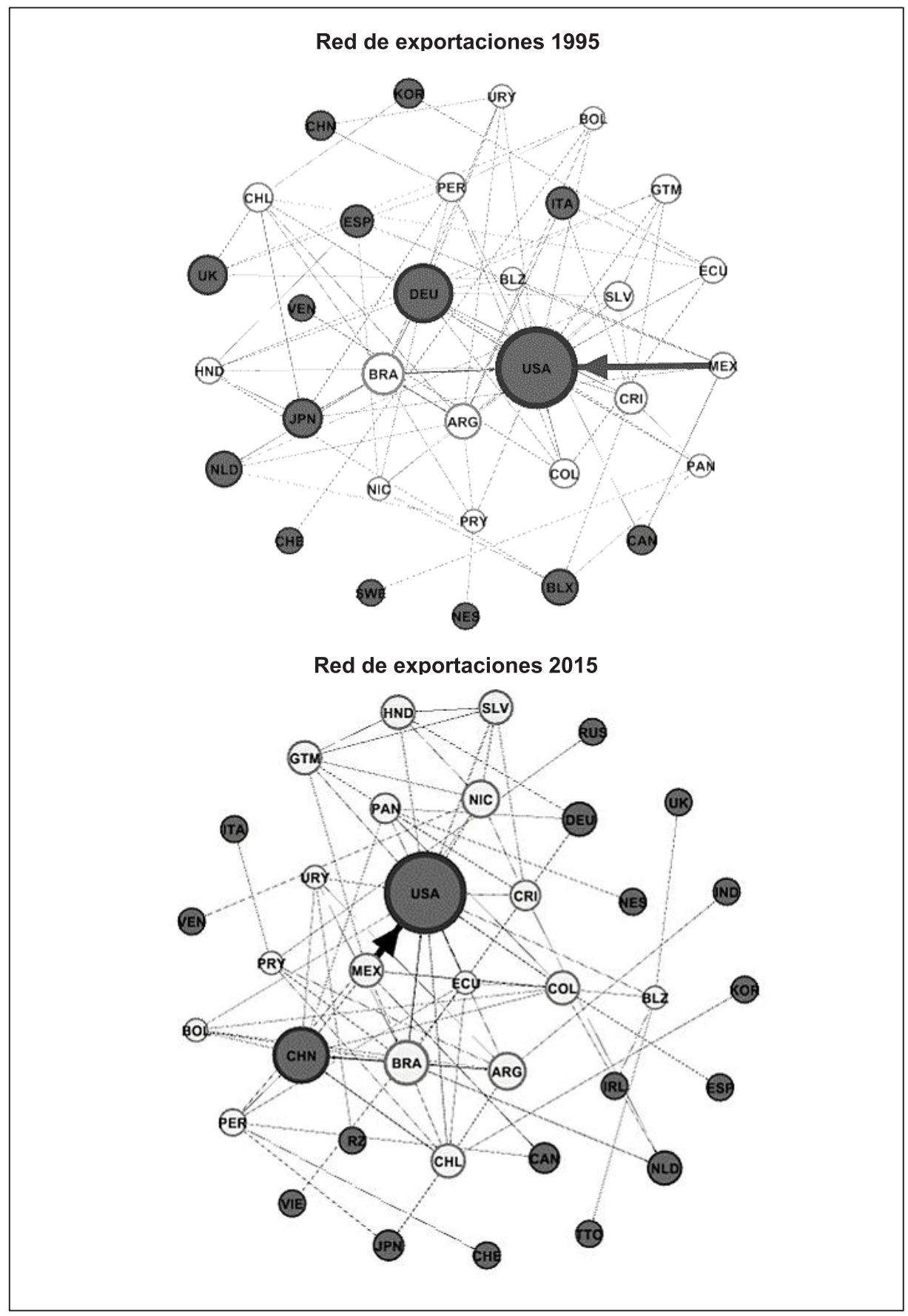

Fuente: elaboración propia utilizando Gephi 0.9.1. 
Guatemala, el segundo socio de la región en términos del volumen de importaciones. Una característica similar en las redes de importaciones y exportaciones es la tendencia a comerciar con economías grandes. El coeficiente de correlación del grado de salida con el PIB es de 0,81 y de 0,85 en 1995 y 2015, respectivamente, lo que implica que en ambos períodos los países latinoamericanos importan desde países con una mayor capacidad productiva.

Tabla 3. Estadísticas descriptivas de las redes de importaciones en 1995 y 2015

\begin{tabular}{|l|c|c|}
\hline \multicolumn{1}{|c|}{ Medida } & 1995 & 2015 \\
\hline Número de nodos & 29 & 26 \\
\hline Diámetro de la red & 3 & 3 \\
\hline Longitud del camino promedio & 1,594 & 1,526 \\
\hline Densidad del grafo & 0,105 & 0,131 \\
\hline Grado promedio & 5,862 & 6,538 \\
\hline Grado ponderado con pesos (valores de las importaciones) & $4.919,034$ & $22.659,962$ \\
\hline Coeficiente de clustering promedio & 0,210 & 0,261 \\
\hline
\end{tabular}

Fuente: elaboración propia utilizando Gephi 0.9 .1

Los cambios ocurridos en la red entre 1995 y 2015 se pueden visualizar en el gráfico 2. Nuevamente se asignó un color claro a los países objeto de estudio y un color oscuro a los socios por fuera de la región. El tamaño de los nodos está determinado por el grado de salida, mientras que el tamaño de las aristas depende del valor monetario del flujo de importaciones, medido en millones de dólares corrientes.

Como se observa, en 1995 Estados Unidos, Guatemala y Japón eran los países más importantes en la red por su centralidad y grado de salida. En 2015, aunque Estados Unidos mantuvo su papel como nodo central en la estructura, se destaca el crecimiento significativo de China como uno de los orígenes más importantes de las importaciones latinoamericanas. En concreto, en 1995 China no estaba dentro de los 5 primeros socios comerciales en importaciones de ningún país y, por tanto, no aparecía en la red; sin embargo, en tan solo 20 años el gigante asiático se convirtió en una de las principales fuentes de importaciones en toda la región, excepto para Guatemala. La consecuencia del posicionamiento de China fue la pérdida de participación en el mercado latinoamericano de países como Guatemala y Japón. 
Gráfico 2. Red de importaciones en 1995 y 2015

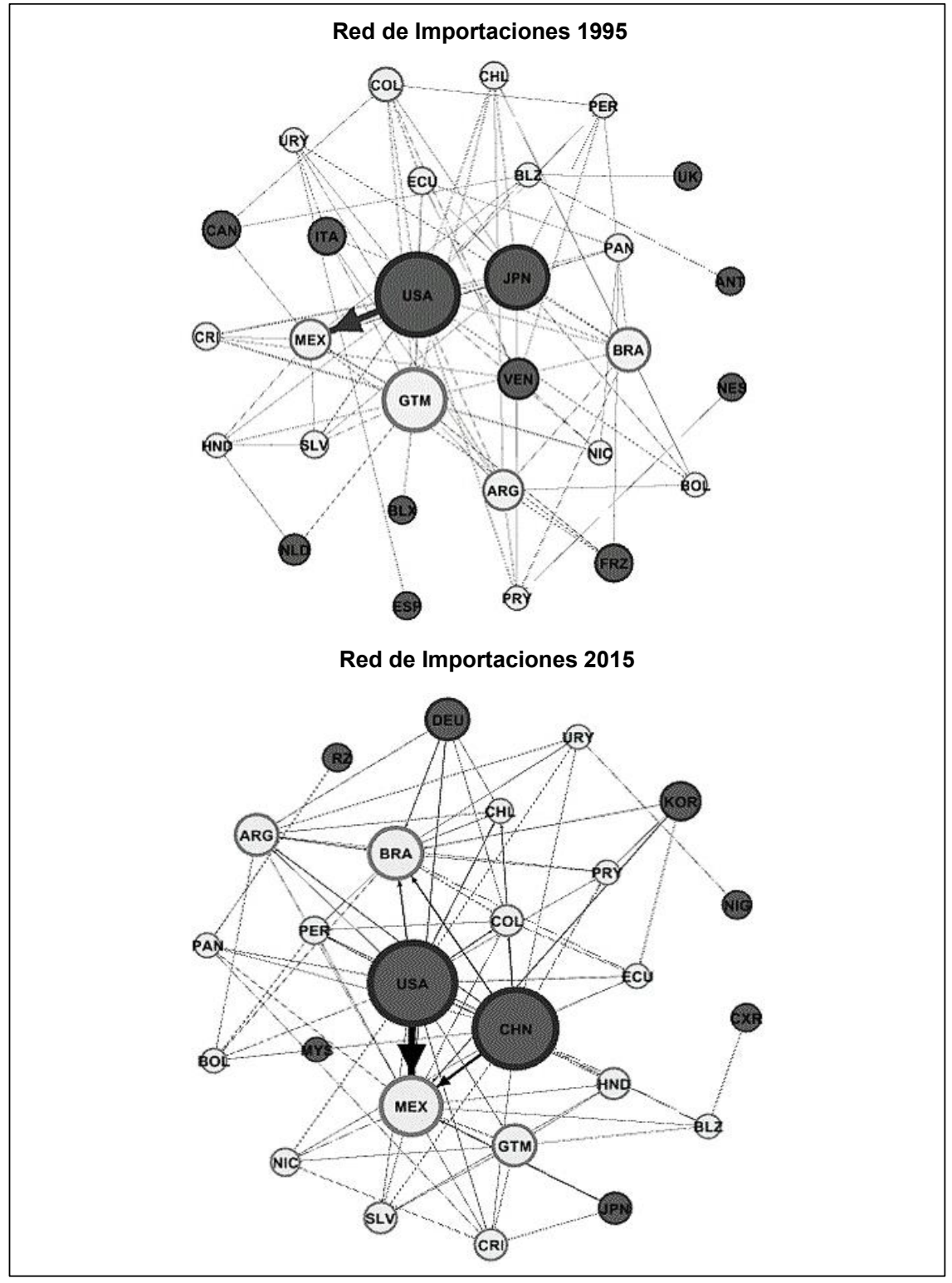

Fuente: elaboración propia utilizando Gephi 0.9.1

En el análisis individual, la centralidad de los nodos en una red ponderada depende no solo del número de conexiones (grado de salida) sino también del valor monetario de las importaciones. En este sentido, Estados Unidos, aunque solo tiene una conexión más que China en 2015, se ubica en una posición más central debido a 
que el grado de salida ponderado es superior en cerca del 70\%. Vale la pena anotar que la centralidad de Estados Unidos está jalonada por el volumen de comercio con México y Guatemala, lo que se evidencia por el tamaño del vínculo que los conecta.

\subsection{Discusión de los resultados}

Los cambios observados en las relaciones comerciales en 1995 y 2015 han estado influenciados por la evolución del panorama económico mundial; de ahí la relevancia de analizar los resultados a la luz de los eventos históricos. Según Durán y Pellandra (2017), uno de los hechos más relevantes en las relaciones económicas internacionales en este siglo es el posicionamiento de China como uno de los actores más importantes en el comercio mundial. Como referencia Perrotti (2015), el país asiático, hoy primer exportador e importador del mundo, alcanzó en este período tasas de crecimiento anual del PIB de dos dígitos, incrementando su PIB per cápita y favoreciendo la salida de la pobreza de más de 500 millones de personas, con un efecto notable sobre los niveles de consumo mundial y los términos de intercambio de muchos productos.

El cambio estructural en la jerarquía del comercio mundial tuvo efectos importantes sobre la estructura productiva de América Latina. Según Jenkins (2011), el incremento del ingreso, el paso de la producción de bienes intensivos en trabajo a sectores más intensivos en capital y el acelerado crecimiento del sector construcción e infraestructura en China generó un fuerte cambio en la demanda y los precios internacionales de los bienes primarios (minerales y metales), fenómeno conocido como el boom de los commodities. En efecto, Coremberg (2012) estima que desde el $2002^{6}$ los precios de las materias primas se incrementaron hasta en $300 \%$, lo cual trajo beneficios sustanciales para los países latinoamericanos ricos en recursos naturales, quienes aumentaron los volúmenes de exportaciones y pudieron disfrutar de un mayor crecimiento económico, amortiguando así los efectos de la crisis financiera del Atlántico Norte iniciada en 2008. Esto explica por qué China se convirtió en socio comercial importante, especialmente para Sudamérica.

Los intercambios se han caracterizado por exportaciones de productos primarios y minerales por parte de América Latina (como soya, metales y petróleo), mientras que las importaciones se han concentrado en bienes manufacturados, como muestra Cepal (2015a). Al respecto, Cepal (2015b) indica que en 2013 el 73\% de las exportaciones totales a China estuvieron concentradas en 5 productos principales: petróleo, hierro, cobre, soja chatarra y madera, mientras en 2000 representaron

6 El incremento de los precios de los productos primarios exportados en la región también puede asociarse con el crecimiento de la demanda de otros países emergentes y la demanda de biocombustibles. 
47\%. Se presenta entonces, un patrón de comercio de tipo centro-periferia, donde China aparece como un nuevo centro y los países latinoamericanos como la periferia, como señala Perrotti (2015).

En los últimos años se ha discutido el efecto mixto de China sobre América Latina, y sus implicaciones en términos de ganadores y perdedores. Como sugiere Cepal (2015a), existe un efecto diferenciado especialmente entre América del Sur y Centroamérica, el Caribe y México. América del Sur, rica en recursos naturales (con excepción de Paraguay), resultó más beneficiada por el mejoramiento en los términos de intercambio y el consecuente incremento de sus ingresos. Sin embargo, estos países experimentaron la llegada de Inversión Extranjera Directa (IED) dirigida al sector primario, que incentivó una reorientación de las actividades productivas y un proceso de desindustrialización. En contraste, Centroamérica, el Caribe y México perdieron mercado para sus exportaciones de textiles y productos manufacturados por el incremento de la producción china; además, se vieron afectados por ser demandantes netos de recursos naturales como el petróleo que sufrieron un incremento en el precio. Esto significa que mientras los denominados perdedores perdieron mercado para sus manufacturas, los ganadores experimentaron una tendencia hacia la producción de productos primarios en su estructura productiva que los hizo más frágiles ante choques externos.

La alta dependencia de la región de los precios y volúmenes de importación de China preocupa porque, como afirman Rosales y Kuwayama (2012), el país asiático ha diversificado sus fuentes de abastecimiento y ahora la región compite en el suministro de los productos primarios y minerales con otros países como Australia, Canadá, Estados Unidos y Nueva Zelandia. Siguiendo a Durán y Pellandra (2017), a pesar de sostener el crecimiento de muchas economías latinoamericanas durante el período de análisis, la relación comercial con China no ha contribuido al desarrollo regional de largo plazo. Además, es importante anotar que a pesar de su importancia para las relaciones comerciales en la región, solo México, Costa Rica, Perú y Chile han establecido tratados comerciales con este país.

Estados Unidos continúa siendo un socio comercial estratégico para la región; su importancia en las relaciones comerciales medida a partir de las conexiones en el gráfico construido, no ha cambiado de forma significativa entre 1995 y 2015. Más allá de su rol como principal destino de exportaciones y fuente de importaciones de la región, es el principal mercado de los bienes manufacturados, y la composición de la canasta exportadora es más diversificada en comparación con China o la Unión Europea. En 2013 los productos primarios y las manufacturas basadas en recursos naturales representaron cerca del $40 \%$ de los envíos totales a Estados 
Unidos. Según datos de Cepal (2015b), el 60\% restante corresponde a manufacturas de tecnología baja, media y alta, producidas principalmente en México y otros países centroamericanos.

Es necesario destacar que, aunque el número de conexiones de Estados Unidos dentro de la red de exportaciones lo mantiene como el nodo principal, su participación en el total de las exportaciones de la región pasó del 58\% en el año 2000 al 40\% en 2010 como indica Cepal (2015b). Esto implica que el comercio con Estados Unidos no ha sido tan dinámico en los años analizados como para contrarrestar las tendencias de mayor producción de bienes primarios y desindustrialización temprana en la región, pues su influencia en la red no se ha acercado a la trascendencia que ha ganado China durante los años de estudio. Por su parte, la participación de la Unión Europea como socio comercial de la región no tuvo un cambio significativo: Rosales (2015) muestra que entre 2000 y 2010, la participación de esta región en el flujo de exportaciones pasó de 12\% al 13\% y la composición de la canasta exportadora, centrada en bienes primarios y manufacturas basadas en recursos naturales, tampoco cambió.

Respecto a las relaciones intrarregionales, llama la atención que a pesar de los múltiples acuerdos comerciales internos, cerca del 30\% de los países no son principales socios de importaciones de sus vecinos: Belice, Nicaragua, Chile, Bolivia, Uruguay, Paraguay. Es interesante notar que a pesar de ser parte del Mercosur, estos últimos no son principales socios de Brasil y Argentina, aunque parte importante de sus importaciones proviene de estos países. Brasil es el principal socio de importaciones de Argentina, mientras que Argentina es el tercer socio de importaciones de Brasil. Con excepción de Brasil, ningún otro país de América Latina tiene un rol relevante como socio de exportaciones en 2015.

\section{COMENTARIOS FINALES}

El análisis de las características topológicas de las redes de comercio en América Latina muestra la importancia que ganó China como socio comercial para todos los países de la región, fundamentalmente de importaciones. Por sus conexiones con todos los países del análisis y con las economías más grandes, así como su centralidad en ambas redes de comercio, China se convirtió en un período de 20 años en el país con mayor influencia para el comercio internacional en Latinoamérica.

Este cambio fue decisivo en la reconfiguración de la estructura económica de los países objeto de análisis, porque mientras el incremento en las importaciones desde China se dio en bienes manufacturados, el crecimiento relativamente me- 
nor de las exportaciones hacia el gigante asiático fue en bienes primarios, sin valor agregado e impulsado por el boom de los commodities. A pesar de esto, solo México, Costa Rica, Perú y Chile tienen acuerdos comerciales con China.

No obstante los esfuerzos y los múltiples acuerdos intrarregionales, no se han podido consolidar relaciones comerciales fuertes. Esto se refuerza con el análisis de las estadísticas descriptivas de las redes, según el cual, no ha sido posible consolidar la conectividad comercial intrarregional, mientras el comercio con otras regiones se ha dinamizado durante el período de análisis. Esto, sumado a la correlación entre el PIB y el grado de entrada y salida en cada red, pone en entredicho la habilidad de los tratados comerciales para jalonar las exportaciones de sus socios más pequeños y evidencia la necesidad de fortalecer los vínculos comerciales internos, lo que permitiría diversificar la producción, aprovechar los acuerdos comerciales ya establecidos y reducir la vulnerabilidad ante choques externos.

Estos cambios reflejan dos problemas de política comercial que deben ser atendidos en el corto plazo. Primero, un problema profundo y que data de mucho antes de que se popularizara la liberalización comercial en América Latina: los procesos de industrialización no han sido fuertes y la inversión en investigación y desarrollo es muy baja, lo que genera relaciones de comercio internacional basadas en intercambios de productos primarios sin valor agregado. Es importante anotar que tener una economía basada en recursos naturales no es un obstáculo, pero sí lo es la baja tecnificación del sector, la poca investigación y la carencia de valor agregado.

Ejemplo de esto es Estados Unidos, que tuvo una economía basada en minerales en el período en que se convirtió en el líder manufacturero del mundo (18901910). Se puede decir que el sector mineral constituyó una fuerza líder en la economía del conocimiento en Estados Unidos y más adelante, el país se convirtió en un líder en la producción de petróleo como afirman Wright y Czelusta (2004). Sin embargo, para que el crecimiento del sector primario esté ligado a un mayor desarrollo económico es necesario un marco legal que lo favorezca y una fuerte inversión en educación e investigación. No son suficientes los subsidios o las reducciones de impuestos, porque pueden terminar en manos de los privados y las multinacionales. Con las políticas apropiadas, se puede aprovechar el potencial en recursos naturales de América Latina.

En segundo lugar, la dependencia económica de la región hacia países externos y el proceso de mayor producción de bienes primarios fue influenciada también por una política comercial que favorece acuerdos comerciales que parecen no estar en línea con las necesidades y potencialidades de sus países, de acuerdo con la com- 
posición de su canasta comercial y con sus ventajas comparativas. Es fundamental que los acuerdos comerciales sean pertinentes con la estructura de la economía y que se establezcan con los socios clave como China porque, formulados de forma correcta, pueden actuar como catalizadores para el crecimiento, financiar la innovación y fomentar la diversificación de las exportaciones, promover la movilización de conocimientos entre fronteras, incrementar la capacidad productiva de la fuerza laboral e impulsar una administración más eficiente de los recursos.

Esta investigación abre la puerta a investigaciones futuras que analicen estas mismas propiedades en bloques más pequeños de la región como Mercosur y la Alianza del Pacífico. También se pueden estudiar las redes dinámicas y la evolución de cada red desde 1995 hasta la actualidad por productos exportados e importados; trabajos de este tipo permitirán hacer un análisis más detallado y arrojarán conclusiones y recomendaciones más precisas sobre el comercio internacional en América Latina.

\section{BIBLIOGRAFÍA}

Bhattacharya, K; Mukherjee, G; Saramaki, J; Kaski, K. y Manna, S. S. (2008). The international Trade Network: Weighted network analysis and modelling. En: Journal of Statistical Mechanics: Theory and Experiment, Vol. 2008, febrero, p. 1-10. doi:10.1088/1742-5468/2008/02/P02002.

Bastian, Mathieu, Heymann, Sebastien y Jacomy, Mathieu (2009). Gephi: an open source software for exploring and manipulating networks. International AAAI Conference on Weblogs and Social Media, 2p.

Cepal (2015a). Perspectivas económicas de América Latina 2016: hacia una nueva asociación con China. OECD Publishing, Paris, 241p.

Cepal (2015b). Panorama de la Inserción Internacional de América Latina y el Caribe 2015. La crisis del comercio regional: diagnóstico y perspectivas. Santiago de Chile, 103p.

Coremberg, A. (2012). La productividad de América Latina ante el auge de los productos básicos. En: Cuadernos Económicos de ICE, No. 84, p. 123-153.

De Benedictis, Luca y Tajoli, Lucia (2011). The World Trade Network. En: The World Economy, Vol. 34, No. 8, agosto, p. 1417-1454. DOI: 10.1111/j.1467-9701.2011.01360.x.

Durán, José y Pellandra, Andrea (2017). La irrupción de China y su impacto sobre la estructura productiva y comercial en América Latina y el Caribe. Cepal, Serie Comercio Internacional, No. 131, Santiago de Chile, 89p.

Fagiolo, Giorgio; Reyes, Javier, y Schiavo, Stefano (2007).International Trade and Financial Integration: A weighted Network Analysis. Documents de Travail de Observatoire Francais des Conjonctures Économiques, No. 2007-11, marzo, 20p.

Fagiolo, Giorgio; Reyes, Javier, y Schiavo, Stefano (2008). On the topological properties of the World Trade Web: A weighted Network Analysis. En: Physica A: Statistical Mechanics and its Applications, Vol. 387, No. 15, junio, p. 3868-3873. 
Fruchterman, Thomas y Reingold, Edward (1991). Graph drawing by force-direct placement. En: Software: Practice and Experience, Vol. 21, No. 11, noviembre, p. 1129-1164.

Garlaschelli, Diego y Loffredo, María (2005). Structure and Evolution of the World TradeNetwork. En: Physica A: Statistical Mechanics and its Applications, Vol. 355, No. 1, septiembre, p. 138 144. https://doi.org/10.1016/j.physa.2005.02.075.

Heymann, Daniel; Perazzo, Roberto y Zimmerman, Martín (2013). Economía de Fronteras Abiertas. 1ra Ed., Buenos Aires, Argentina: Teseo, 370p.

Hilgerdt, Folke (1943). The case for multilateral trade. En: The American Economic Review, Vol. 33, No 1, marzo, p. 393-407.

Jackson, Mathew (2008). Social an economic networks. Princeton: Princeton University Press, 520p.

Jenkins, Rhys (2011). The "China effect" on commodity process and Latin American export earnings. En: Revista Cepal, No. 103, abril, p. 73-87.

Kali, Raja y Reyes, Javier (2007). The architecture of globalization: A network approach to international economic integration. En: Journal of International Business Studies, Vol. 38, No. 4, p. 595-620.

Kastelle, Tim; Steen, John y Liesch, Peter (2006). Measurig globalisation: An evolutionary economic approach to tracking the evolution of international trade. DRUID Summer Conference on Knowledge, Innovation and Competitiveness: Dynamycs of Firms, Networks, Regions and Institutions, Copenhagen, Denmark, junio 18-20, 41p.

Li, Xiang; Jin, Yu y Chen, Guanrong (2003). Complexity and synchronization of the World trade Web. En: Physica A: Statistical Mechanics and its Applications, Vol. 328, No. 1-2, octubre, p. 287-296. https://doi.org/10.1016/S0378-4371(03)00567-3

Perrotti, D. (2015). La República Popular de China y América Latina: impacto del crecimiento económico chino en las exportaciones latinoamericanas. En: Revista Cepal, No. 116, p. 47-60.

Rosales, Osvaldo (2015). América Latina y el Caribe y China. Hacia una nueva era de cooperación Económica. Santiago de Chile, Chile: Naciones Unidas, 81p.

Rosales, Osvaldo y Kuwayama, Mikio (2012). China y América Latina y el Caribe: hacia una relación económica y comercial estratégica. Santiago de Chile, Cepal: Publicación de las Naciones Unidas, 252p.

Serrano, Angeles y Boguña, Marián (2003). Topology of the world trade web. En: Physical Review E, Vol. 68, julio, 015101 (R). DOI:https://doi.org/10.1103/PhysRevE.68.015101.

Serrano, Angeles y Boguña, Marián y Vespignani, Alessandro (2007). Patterns of dominant flows in the world trade web. En: Journal of Economic Interaction and Coordination, Vol. 2, No. 2, p. 111-124. DOI: 10.1007/s11403-007-0026-y.

Smith, David y White, Douglas (1992). Structure and Dynamics of the Global Economy: Network Analysis of International Trade 1965-1980. En: Social Forces, Vol. 70, No.4, p. 857-93. 
Vega Redondo, Fernando (2007). Complex Social Networks. Nueva York: Cambridge University Press, 312p.

Wright, Gavin y Czelusta, Jesse (2004). The Myth of the Resource Curse. En: Challenge, Vol. 47, No. 2, marzo-abril, p. 6-38.

Zhang, Xiaohang; Huiyuan, Cui; Zhu, Ji; Du, Yu; Wang, Oi y Shi, Wenhua (2017). Measuring the dissimilarity of multiplex networks: An empirical study of international trade networks. En: Physica A: Statistical Mechanics and its Applications, Vol. 467, febrero, p. 380-396. 


\section{ANEXO}

Tratados comerciales de los países latinoamericanos, vigentes en 2016

\begin{tabular}{|l|c|c|l|c|c|l|c|c|}
\hline \multicolumn{1}{|c|}{ País } & $\begin{array}{c}\text { Tratados } \\
\text { Internos }\end{array}$ & $\begin{array}{c}\text { Tratados } \\
\text { Externos }\end{array}$ & \multicolumn{1}{|c|}{ País } & $\begin{array}{c}\text { Tratados } \\
\text { Internos }\end{array}$ & $\begin{array}{c}\text { Tratados } \\
\text { Externos }\end{array}$ & País & $\begin{array}{c}\text { Tratados } \\
\text { Internos }\end{array}$ & $\begin{array}{c}\text { Tratados } \\
\text { Externos }\end{array}$ \\
\hline México & 13 & 6 & Costa Rica & 8 & 7 & Bolivia & 5 & 0 \\
\hline Belice & 4 & 1 & Panamá & 6 & 7 & Chile & 11 & 15 \\
\hline Guatemala & 9 & 3 & Colombia & 11 & 4 & Argentina & 9 & 4 \\
\hline Honduras & 8 & 3 & Venezuela & 12 & 2 & Uruguay & 8 & 3 \\
\hline El Salvador & 7 & 4 & Ecuador & 5 & 0 & Paraguay & 7 & 3 \\
\hline Nicaragua & 8 & 4 & Perú & 7 & 9 & Brasil & 10 & 3 \\
\hline
\end{tabular}

Fuente: elaboración propia con datos de SICE y Aladi 